\title{
MESSENGER observations of large flux transfer events at Mercury
}

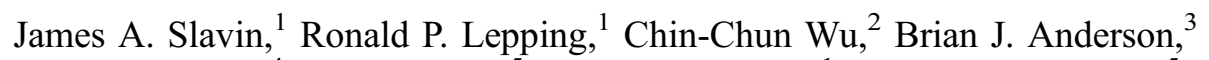 \\ Daniel N. Baker, ${ }^{4}$ Mehdi Benna, ${ }^{5}$ Scott A. Boardsen, ${ }^{1}$ Rosemary M. Killen, ${ }^{5}$ Haje Korth, ${ }^{3}$ \\ Stamatios M. Krimigis, ${ }^{3,6}$ William E. McClintock, ${ }^{4}$ Ralph L. McNutt Jr., ${ }^{3}$ \\ Menelaos Sarantos, ${ }^{1}$ David Schriver, ${ }^{7}$ Sean C. Solomon, ${ }^{8}$ Pavel Trávníček, ${ }^{9}$ \\ and Thomas H. Zurbuchen ${ }^{10}$
}

Received 23 October 2009; revised 8 December 2009; accepted 15 December 2009; published 29 January 2010.

[1] Six flux transfer events (FTEs) were encountered during MESSENGER's first two flybys of Mercury (M1 and M2). For M1 the interplanetary magnetic field (IMF) was predominantly northward and four FTEs with durations of 1 to $6 \mathrm{~s}$ were observed in the magnetosheath following southward IMF turnings. The IMF was steadily southward during M2, and an FTE $4 \mathrm{~s}$ in duration was observed just inside the dawn magnetopause followed $\sim 32$ s later by a 7-s FTE in the magnetosheath. Flux rope models were fit to the magnetic field data to determine FTE dimensions and flux content. The largest FTE observed by MESSENGER had a diameter of $\sim 1 R_{\mathrm{M}}$ (where $R_{\mathrm{M}}$ is Mercury's radius), and its open magnetic field increased the fraction of the surface exposed to the solar wind by 10-20 percent and contributed up to $\sim 30 \mathrm{kV}$ to the cross-magnetospheric electric potential. Citation: Slavin, J. A., et al. (2010), MESSENGER observations of large flux transfer events at Mercury, Geophys. Res. Lett., 37, L02105, doi:10.1029/2009GL041485.

\section{Introduction}

[2] The MErcury Surface, Space ENvironment, GEochemistry, and Ranging (MESSENGER) flyby measurements show that Mercury's magnetic field is largely dipolar, has a moment closely aligned with the planet's rotation axis with the same polarity as at Earth, and has not significantly changed since its discovery by Mariner 10 in 1974 and 1975 [Anderson et al., 2008, 2009; I. I. Alexeev et al., Mercury's magnetospheric magnetic field after the first two MESSENGER flybys, submitted to Icarus, 2009]. The

\footnotetext{
${ }^{1}$ Heliophysics Science Division, NASA Goddard Space Flight Center, Greenbelt, Maryland, USA.

${ }^{2}$ Naval Research Laboratory, Washington, D. C., USA.

${ }^{3}$ Johns Hopkins University Applied Physics Laboratory, Laurel, Maryland, USA.

${ }^{4}$ Office of Space Research and Technology, Academy of Athens, Athens, Greece.

${ }^{5}$ Laboratory for Solar and Atmospheric Physics, University of Colorado, Bounder, Colorado, USA.

${ }^{6}$ Solar System Exploration Division, NASA Goddard Space Flight Center, Greenbelt, Maryland, USA.

${ }^{7}$ Institute for Geophysics and Planetary Physics, University of California, Los Angeles, California, USA.

${ }^{8}$ Department of Terrestrial Magnetism, Carnegie Institution of Washington, Washington, D. C., USA.

${ }^{9}$ Astronomical Institute, Academy of Sciences of the Czech Republic, Prague, Czech Republic.

${ }^{10}$ Department of Atmospheric, Oceanic and Space Sciences, University of Michigan, Ann Arbor, Michigan, USA.
}

Copyright 2010 by the American Geophysical Union. 0094-8276/10/2009GL041485\$05.00 interaction of the planetary magnetic field with the solar wind is governed primarily by the interplanetary magnetic field (IMF) orientation. For the first MESSENGER Mercury flyby (M1) on 14 January 2008 the average IMF upstream of the outbound bow shock was northward with $\left(B_{\mathrm{X}}, B_{\mathrm{Y}}\right.$, $\left.B_{\mathrm{Z}}\right)=(-12.9,4.71,10.29 \mathrm{nT})$ in Mercury solar orbital (MSO) coordinates. In these coordinates $X_{\mathrm{MSO}}$ is directed from the center of the planet toward the Sun, $Z_{\mathrm{MSO}}$ is normal to Mercury's orbital plane and positive toward the north celestial pole, and $Y_{\text {MSO }}$ completes this right-handed orthogonal system. In contrast, for MESSENGER's second Mercury flyby (M2) on 6 October 2008, the mean upstream IMF was southward, $\left(B_{\mathrm{X}}, B_{\mathrm{Y}}, B_{\mathrm{Z}}\right)=(-15.21,8.40,-8.51 \mathrm{nT})$.

[3] Magnetic reconnection occurs at the dayside magnetopause when there is a component of the IMF anti-parallel to the subsolar magnetospheric magnetic field. When such reconnection is localized or non-steady at Earth, discrete magnetic flux tubes with diameters of $\sim 1 R_{\mathrm{E}}$ (where $1 R_{\mathrm{E}}=$ $6400 \mathrm{~km}$ ), termed flux transfer events (FTEs), become connected to the IMF and are pulled from the dayside magnetosphere by the anti-sunward flow in the magnetosheath and added to the tail [Russell and Elphic, 1978]. FTEs created by reconnection occurring simultaneously at multiple dayside X-lines are identified by their flux rope structure [Lee and Fu, 1985]. FTEs not possessing flux rope topology may be produced by short-duration pulses of reconnection [Southwood et al., 1988; Scholer, 1988]. They are identified primarily by the characteristic manner in which magnetosheath and magnetospheric magnetic fields drape about these flux tubes as they move tailward.

[4] Some FTEs were found and analyzed in the Mariner 10 flyby observations [Russell and Walker, 1985], and initial examinations of the MESSENGER magnetic field measurements also noted the presence of FTEs [Slavin et al., 2008, 2009b]. Here we report a comprehensive survey of the MESSENGER magnetic field data for the occurrence of FTEs. From definitions developed for Earth's magnetosphere [e.g., Wang et al., 2005; Fear et al., 2007], six FTEs were identified during the two flybys with all, save one, strongly resembling flux ropes. Unfortunately, MESSENGER does not make the high-time-resolution plasma moment measurements necessary to analyze these FTEs using the Grad-Shafranov (GS) reconstruction technique [Zhang et al., 2008; Eriksson et al., 2009]. However, we use a well validated flux rope model [Lepping et al., 1990, 2006] to infer their dimensions and orientation, the proximity of the spacecraft path to the rope's central axis, and their axial magnetic flux content. In contrast with the Mariner 10 findings, the MESSENGER results indicate that some FTEs 


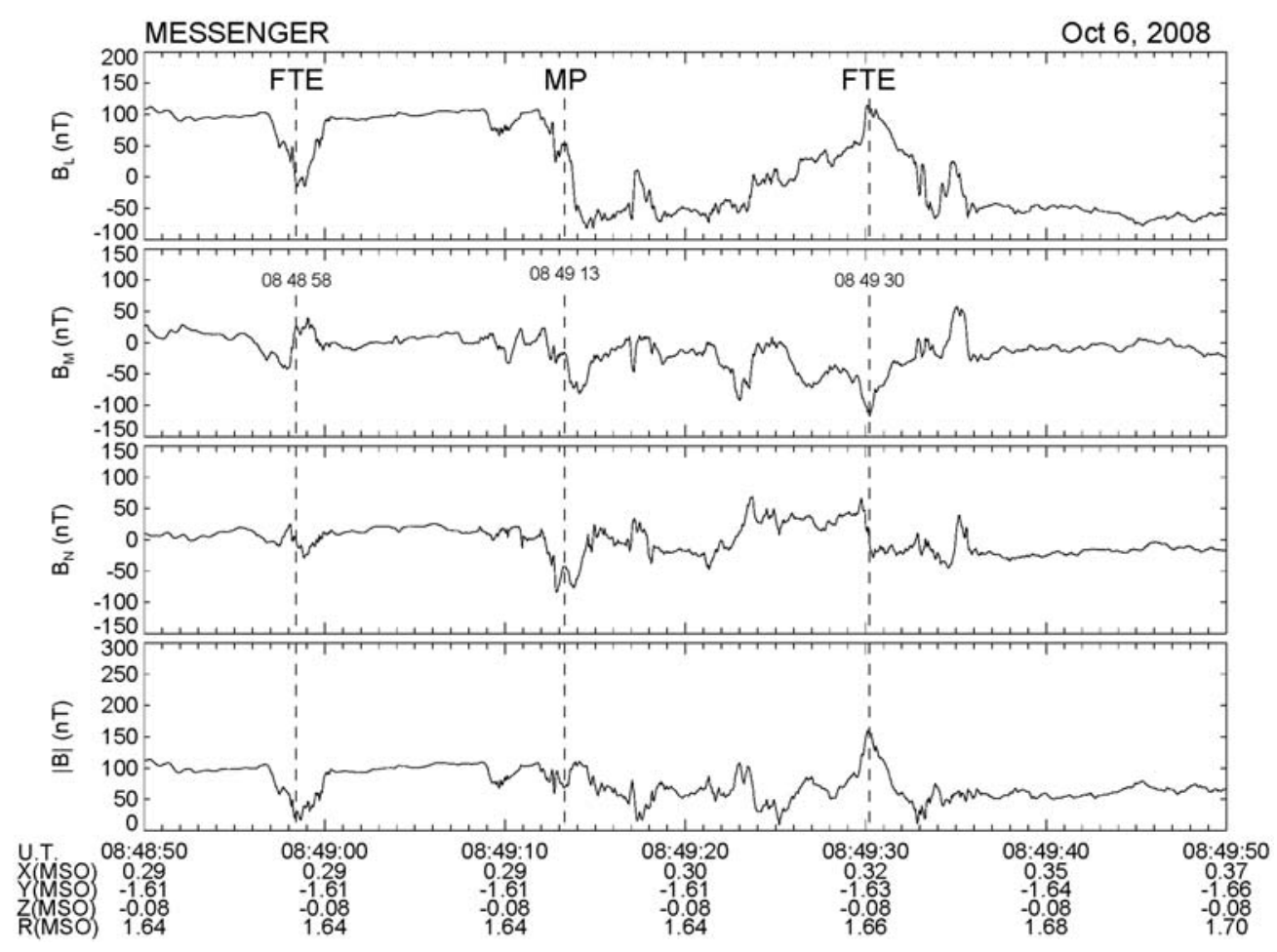

Figure 1. MESSENGER magnetic field measurements across the M2 dawn magnetopause in boundary-normal coordinates.

at Mercury carry as much flux as typical FTEs at Earth. It is concluded that these large FTE's will have significant impacts on the cross-magnetospheric electric potential drop and the flux of solar wind ions reaching the surface and sputtering neutral atoms into Mercury's exosphere.

\section{MESSENGER Flux Transfer Event Observations}

[5] Near the magnetopause, FTEs are identified by variations of the magnetic field in a local boundary-normal coordinate system [Russell and Elphic, 1978]. We present data in L-M-N coordinates, where $B_{\mathrm{N}}$ is directed radially outward normal (using the Slavin et al. [2009a] model) to the closest point on the magnetopause, $B_{\mathrm{L}}$ is perpendicular to $B_{\mathrm{N}}$ and anti-parallel to the planetary magnetic dipole, and $B_{\mathrm{M}}$ completes the right-handed system.

[6] We identify two M2 FTE bipolar $B_{\mathrm{N}}$ signatures in Figure 1, the first lasting $3.5 \mathrm{~s}$ at 08:48:58 UTC and the second lasting 7.1 s at 08:49:30. The sense of the bipolar $B_{\mathrm{N}}$ variation for both FTEs is consistent with reconnection occurring at a tilted X-line passing near the subsolar point and moving northward over MESSENGER. The decrease in magnetic field intensity within the 08:48:58 event is very similar to "crater-type" FTEs at Earth. The crater feature is thought to correspond to a "swirl" of plasma with a high ratio of magnetic to kinetic pressure caused by ongoing reconnection [Owen et al., 2008]. The second event at 08:49:30 is the longest-duration FTE found in the M1 and M2 data and exhibits a strong core magnetic field and helical topology, evident in $B_{\mathrm{L}}$ and $B_{\mathrm{M}}$, typical of a quasiforce-free flux rope. In this event the core magnetic field exceeds the surrounding magnetosheath field by a factor of $\sim 2.5$.

[7] Another long-duration FTE lasting $6 \mathrm{~s}$ was observed during M1 inbound near the dusk flank. Figure 2 shows data both for this event on the left and for the 7-s FTE discussed above on the right, here presented in MSO coordinates. Vertical dashed lines mark the beginning and end points of each event estimated from the field rotational signature. In each case the flux-rope-like variation in the magnetic field is evident in the rotational signature surrounding an enhancement in the total field. The magnetic field magnitude and rotation in Figure 2a are nearly symmetric relative to the time of maximum field intensity, whereas the FTE in Figure $2 b$ has a narrow, somewhat asymmetric field magnitude enhancement relative to the field rotation. Both of the FTEs are associated with an IMF $B_{\mathrm{Z}}<0$ in the magnetosheath, as occurred intermittently inbound for M1, but nearly continuously inbound and outbound for M2. Our examination of the MESSENGER magnetic field data revealed three additional magnetosheath FTEs during M1, which are displayed in Figure 3. These FTEs were also associated with magnetosheath $B_{\mathrm{Z}}<0$, although there is a brief (less than $1 \mathrm{~min}$ ) period of northward magnetic field separating the FTE in Figure 3c from the end of the earlier interval of southward IMF. These FTEs were shorter, lasting $\sim 1 \mathrm{~s}$ to $3 \mathrm{~s}$, but they all have magnetic field perturbations similar to those of the longer-duration events.

\section{Force-Free Modeling of Flux Transfer Events}

[8] We investigate the structure of the FTEs observed by MESSENGER in Mercury's magnetosheath by modeling 


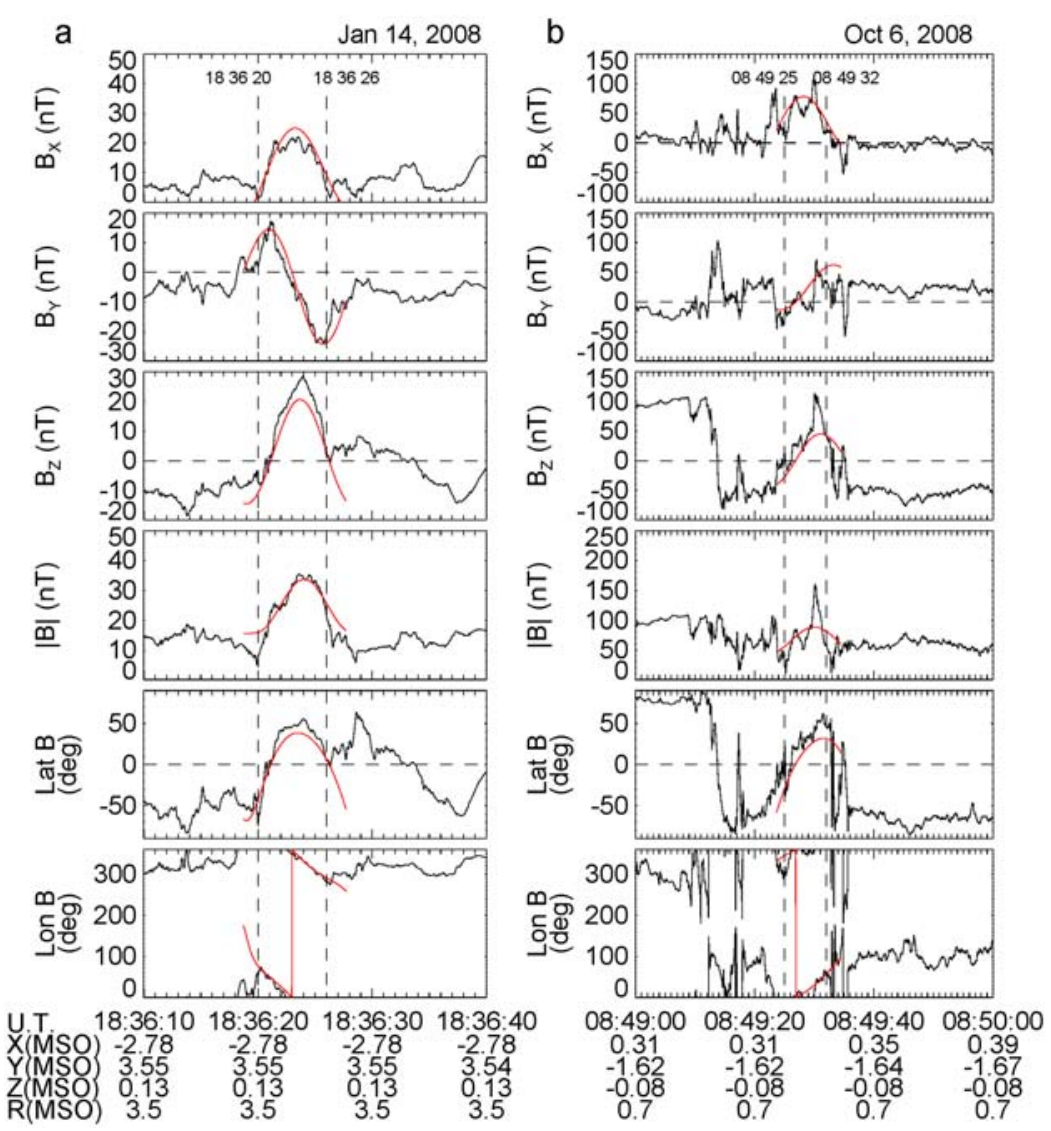

Figure 2. Magnetic field measurements in MSO coordinates for the largest FTEs identified during (a) M1 and (b) M2. Force-free flux rope models fit to these events are shown in red. Dashed vertical lines mark the selected beginning and end of the fitting interval.
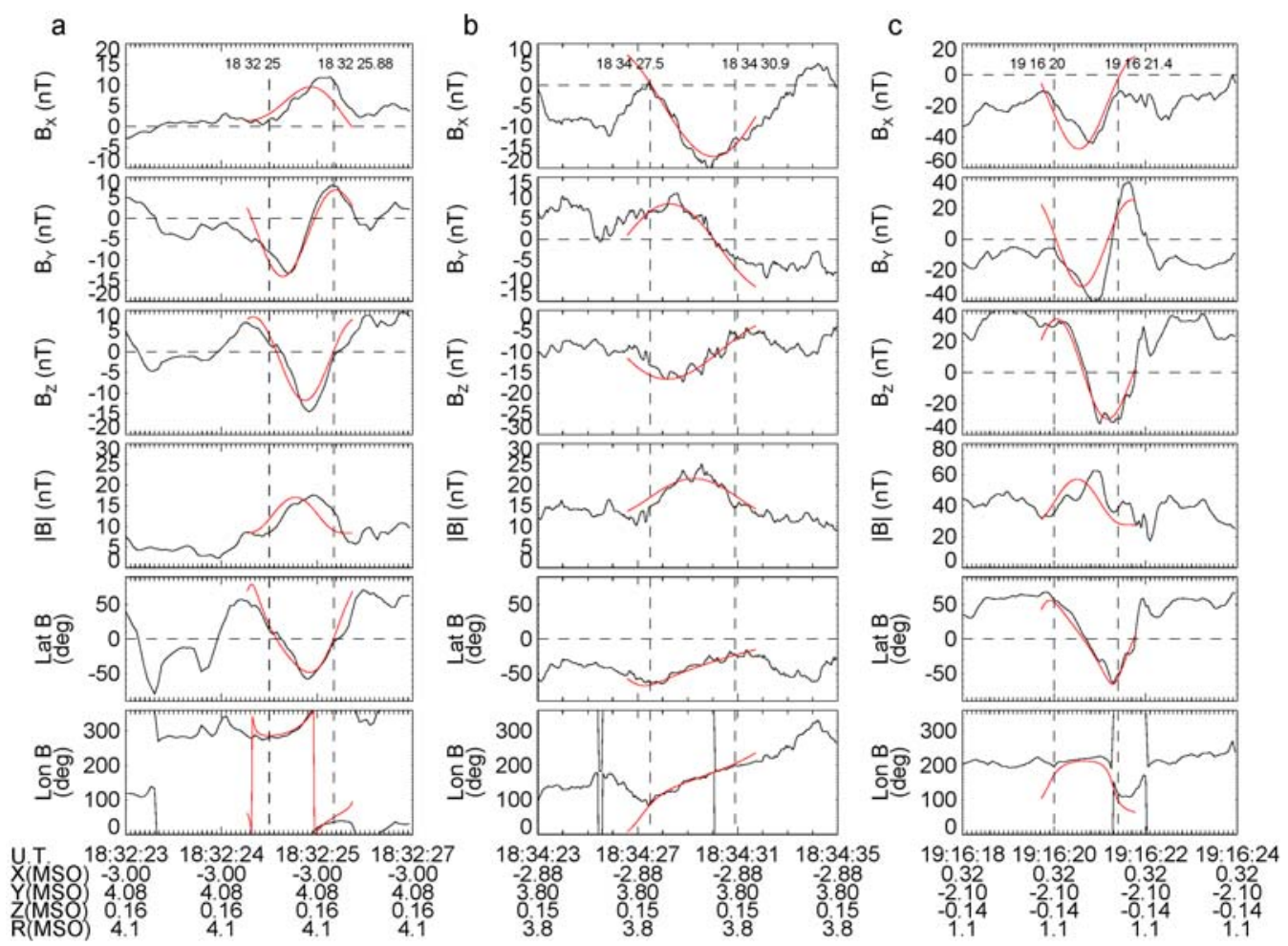

Figure 3. Magnetic field measurements of FTEs observed during M1 with constant- $\alpha$ flux rope models shown in dark red. 
Table 1. Flux Transfer Event Modeling Results

\begin{tabular}{ccccccccccccc}
\hline Event & DOY & Start Time (UTC) & Duration (s) & $Q_{\chi}$ & ASF & $H^{\mathrm{a}}$ & $R_{0}{ }^{\mathrm{b}}\left(R_{\mathrm{M}}\right)$ & $\left|Y_{0} / R_{0}\right|$ & $B_{0}(\mathrm{nT})$ & $\theta_{\mathrm{A}}(\mathrm{deg})$ & $\varphi_{\mathrm{A}}(\mathrm{deg})$ & $\Phi(\mathrm{MWb})$ \\
\hline 1 & 014 & $18: 32: 24$ & 0.97 & 0.101 & 0.12 & $\mathrm{~L}$ & 0.078 & 0.53 & 20.9 & -53.9 & 254.9 & 0.0011 \\
2 & 014 & $18: 34: 27$ & 3.42 & 0.049 & 0.055 & $\mathrm{~L}$ & 0.35 & 0.69 & 30.3 & 5.7 & 132.3 & 0.030 \\
3 & 014 & $18: 36: 20$ & 6.00 & 0.082 & 0.202 & $\mathrm{~L}$ & 0.52 & 0.46 & 38.7 & 69.8 & 302.9 & 0.085 \\
4 & 014 & $19: 16: 19$ & 1.37 & 0.169 & 0.000 & $\mathrm{~L}$ & 0.086 & 0.00 & 57.5 & 12.8 & 228.9 & 0.0035 \\
5 & 280 & $08: 49: 25$ & 7.09 & 0.140 & 0.169 & $\mathrm{R}$ & 0.49 & 0.52 & 108.2 & 58.0 & 354.8 & 0.22 \\
\hline
\end{tabular}

${ }^{\mathrm{a}} H$ is handedness: $\mathrm{R}$ for right-handed and $\mathrm{L}$ for left-handed.

${ }^{\mathrm{b}} V=400 \mathrm{~km} / \mathrm{s}$ is assumed for all cases except event 5 , for which $V=250 \mathrm{~km} / \mathrm{s}$.

them as force-free flux ropes [Lepping et al., 1990]. Originally developed for interplanetary magnetic clouds, this procedure has also been applied to a variety of flux ropes in Earth's magnetotail. The model is based on the assumption that the flux rope current density $(\mathbf{J})$ and magnetic field (B) are related by a constant of proportionality, $\alpha$;

$$
\mathbf{J}=\alpha \mathbf{B}
$$

The structure is assumed to be cylindrically symmetric, with the pitch angle of the helical field lines increasing with distance from the axis of the rope. The field at the center of the rope is aligned with its central axis, becoming perpendicular at the outer boundary of the rope. An analytical approximation for this field configuration is the static, constant- $\alpha$, force-free, cylindrically symmetric configuration, a solution to

$$
\nabla^{2} \mathbf{B}=-\alpha^{2} \mathbf{B}
$$

The Lundquist [1950] Bessel function solution is:

$$
B_{z}(r)=B_{0} J_{0}\left(\alpha^{2}\right), B_{\theta}(r)=B_{0} H J_{1}\left(\alpha^{2}\right), \text { and } B_{\mathrm{r}}=0
$$

where $B_{0}$ is the peak axial field intensity and $H= \pm 1$ is the rope's handedness.

[9] Using the method of Lepping et al. [1990, 2006], we fit equation (3) to the measured magnetic field (in MSO coordinates) for all of the flux rope events. The data were first normalized, and then a variance analysis was applied to establish an approximate rope coordinate system. We then performed a least-squares fit between the normalized, observed magnetic field after transformation into this initial coordinate system, and equation (3). Given the orientation of the flux rope relative to the spacecraft trajectory, the radius of the flux rope was inferred from the estimated magnetosheath plasma flow speed. A flow speed of $250 \mathrm{~km} / \mathrm{s}$ was assumed for the one near-magnetopause FTE and $400 \mathrm{~km} / \mathrm{s}$ for the other FTEs, on the basis of numerical simulations of solar wind flow about Mercury's magnetosphere for the flybys [Benna et al., 2009; P. M. Trávníèek et al., Mercury's magnetosphere-solar wind interaction for northward and southward interplanetary magnetic field: Hybrid simulation results, submitted to Icarus, 2009].

[10] Several parameters were calculated for each flux-rope fit. A "reduced chi" quality parameter, $Q_{\chi}=\chi /(3 N-n)$, was used to measure the quality of the fit, where $\chi$ is the variance of the data relative to the fit, $N$ is the number of points considered in the analysis interval, and $n=5$ is the number of parameters used in the fit. Note that $Q_{\chi}$ is dimensionless since the magnetic field was normalized. A reduced $Q_{\chi}$ of less than 0.25 is required before a fit is regarded as "acceptable" [see Lepping et al., 2006]. The quality of the fit is also judged by the symmetry of the fitted field intensity. We define an asymmetry factor, ASF $=\mid(1-$ $\left.2\left(t_{0} / \Delta t\right) /(N-1)\right) \mid$, where $t_{0}$ is the center time of the rope and $\Delta t$ is the sampling interval. An ASF of 0 is an ideal fit to a force-free cylindrical flux rope, and values over 0.5 are not acceptable. Ideally the field is purely azimuthal (i.e., where $\alpha r=2.4)$ at the flux rope boundary, but in practice the precise end points are not always evident in the data. For this reason trial fits are generally necessary, with the best fit chosen on the basis of $Q_{\chi}$ and ASF. The flux rope parameters derived from the fits are $B_{0}$, the axial field intensity; $H$, the handedness ( \pm 1 for right/left hand); $R_{0}$, the radius of the flux rope; $Y_{0}$, the closest approach distance of the spacecraft to the rope's axis; $Y_{0} / R_{0}$, the "impact parameter;" $\theta_{\mathrm{A}}$ and $\varphi_{\mathrm{A}}$, the polar and longitude angles of the rope's axis, respectively; and $t_{0}$, the rope center time.

[11] The model fit to the M1 FTE observed at 18:36:20 is displayed in Figure 2a. The best-fit model parameters are given in Table 1 (as event 3 ). The agreement between the data and the flux rope model is excellent. The $Q_{\chi}$ and ASF parameters are small, 0.082 and 0.20 , respectively. The inferred flux rope radius is $0.52 R_{\mathrm{M}}$ (where $R_{\mathrm{M}}$ is Mercury's radius), and $B_{0}$ is $39 \mathrm{nT}$. The spacecraft closest approach distance was halfway out from the central axis, $Y_{0} / R_{0}=$ 0.46 . The polar and longitude angles are $70^{\circ}$ and $303^{\circ}$, respectively, indicating that the rope was highly inclined to the MSO X-Y plane and close to the upstream IMF direction.

[12] The model fit to the M2 high-field-intensity event at $08: 49: 30$ observed just upstream of the magnetopause is shown in Figure 2b, and parameters are listed in Table 1 (as event 5). Although the best-fit model does not reproduce the extreme peak field intensity, the angular variations in the magnetic field direction are well matched. The fit quality factors are acceptable, with $Q_{\chi}=0.140$ and $\mathrm{ASF}=0.17$. The inferred radius of the flux rope is $0.49 R_{\mathrm{M}}$, and the maximum axial magnetic field intensity is $108 \mathrm{nT}$. The spacecraft closest approach distance to the central axis of the flux rope was again about halfway out from the axis with $Y_{0} / R_{0}=0.52$. This flux rope had a latitude angle of $\theta_{\mathrm{A}}=$ $58^{\circ}$, while the longitudinal orientation was sunward at $\varphi_{\mathrm{A}}=$ $355^{\circ}$. The fit results for the three remaining magnetosheath FTEs are graphed in Figure 3, and their fit parameters are listed in Table 1.

\section{Summary and Conclusions}

[13] The MESSENGER FTEs are significantly longer in duration than the $\sim 1$-s FTEs identified from Mariner 10 
data and analyzed by Russell and Walker [1985]. Only two of the six MESSENGER FTEs are less than $2 \mathrm{~s}$ in duration, while the other four have durations of 3.4 to $7.1 \mathrm{~s}$. The reason why the MESSENGER FTEs are larger is unclear, but it may be due to differences in upstream solar wind conditions between the times of the MESSENGER and Mariner 10 flybys. The 32-s interval between the two M2 FTEs is similar to the $\sim 30-40$-s period large-amplitude magnetospheric compressional perturbations reported by Anderson et al. [2009] and the $230-60$-s spacing between the plasmoid and traveling compression regions in the tail found by Slavin et al. [2009a]. The comparability of these periods raises the possibility that the formation and tailward motion of FTEs may produce global compressions of the forward magnetosphere and episodes of reconnection in the tail.

[14] Our modeling indicates that the MESSENGER FTEs can be represented as quasi-force-free flux ropes. Their diameters and axial magnetic flux contents varied from $D=0.15$ to $1.04 R_{\mathrm{M}}$ and $\Phi=0.001$ to $0.2 \mathrm{MWb}$. The largest of the FTEs observed by MESSENGER have diameters that exceed by a factor of $\sim 2$ the mean thickness of the magnetosheath at the local time when they were observed. However, as noted above MESSENGER does not make the high-time-resolution plasma moment measurements that would be required to infer FTE flattening using GS reconstruction techniques. Given their great relative size, the FTEs documented here could be significantly deformed by their interaction with the magnetosheath and the shape and location of magnetopause and bow shock locally altered. By comparison, the typical FTE observed at Earth has a diameter of $\sim 1 R_{\mathrm{E}}$, which is only $\sim 30 \%$ of the mean subsolar magnetosheath thickness at Earth.

[15] Moreover, the axial magnetic flux of the largest MESSENGER FTEs approaches that of FTEs observed at Earth [Zhang et al., 2008, and references therein]. This result suggests that FTE size may be controlled not by the dimensions of the magnetosphere, but by the plasma kinetic properties of the solar wind or the reconnection process as has been previously suggested [Kuznetsova and Zeleny, 1986]. The variation in tail lobe magnetic flux from relatively quiescent, northward IMF, to more active, southward IMF intervals at Earth and Mercury are estimated to be $\sim 500$ to $700 \mathrm{MWb}$ [Huang et al., 2009] and 4 to $6 \mathrm{MWb}$ (Alexeev et al., submitted manuscript, 2009), respectively. Hence, while a large FTE at Earth transports perhaps 0.1\% of the quiet-time lobe flux, the situation at Mercury is quite different, with a large FTE carrying $\sim 5 \%$ of the total lobe flux. The transfer of this magnetic flux from the dayside to the nightside magnetosphere will contribute to the crossmagnetospheric electric potential an amount $\Phi / \Delta T$, where $\Delta T \sim D / 400 \mathrm{~km} / \mathrm{s}$ is the time scale for the flux change to the dawn-to-dusk magnetospheric electric potential. The values range from $\sim 1 \mathrm{kV}$ for the smallest to $\sim 30 \mathrm{kV}$ for the largest MESSENGER FTEs.

[16] The magnetic flux content of the FTEs observed by MESSENGER may also have significant implications for solar wind access to the surface of the planet and, therefore, for the variability in the sputtered component of Mercury's exosphere. For IMF $B_{\mathrm{X}}$ oriented away from the Sun and $B_{\mathrm{Z}} \sim-10 \mathrm{nT}$, i.e., conditions close to those during MESSENGER's second flyby, Sarantos et al. [2007] estimated that
$12 \%$ of the northern hemisphere is magnetically "open" and exposed to the solar wind. Magnetic flux conservation indicates that a $0.2 \mathrm{MWb}$ FTE will expose an additional $\sim 10-20 \%$ of the surface to solar wind impact. However, this newly open magnetic flux will be concentrated in the cusp regions where most of the solar wind ion precipitation occurs [Sarantos et al., 2007]. For this reason FTEs may produce brief increases in solar wind ion impact with amplitudes of many tens of percent relative to the mean cusp precipitation rate and the rate at which neutrals are sputtered into Mercury's exosphere.

[17] Acknowledgments. Computational assistance and data visualization support provided by J. Feggans are gratefully acknowledged. Conversations with S. Imber and A. Glocer are appreciated. The MESSENGER project is supported by the NASA Discovery Program under contracts NASW-00002 to the Carnegie Institution of Washington and NAS5-97271 to the Johns Hopkins University Applied Physics Laboratory.

\section{References}

Anderson, B. J., M. H. Acuña, H. Korth, M. E. Purucker, C. L. Johnson, J. A. Slavin, S. C. Solomon, and R. L. McNutt Jr. (2008), The structure of Mercury's magnetic field from MESSENGER's first flyby, Science, 321, 82 -85, doi:10.1126/science. 1159081

Anderson, B. J., et al. (2009), The magnetic field of Mercury, Space Sci. Rev., doi:10.1007/s11214-009-9544-3, in press.

Benna, M., et al. (2009), Modeling of the magnetosphere of Mercury at the time of the first MESSENGER flyby, Icarus, in press.

Eriksson, S., et al. (2009), Magnetic island formation between large-scale flow vortices at an undulating postnoon magnetopause for northward interplanetary magnetic field, J. Geophys. Res., 114, A00C17, doi:10.1029/2008JA013505.

Fear, R. C., S. E. Milan, A. N. Fazakerley, C. J. Owen, T. Asikainen, M. G. G. T. Taylor, E. A. Lucek, H. Rème, I. Dandouras, and P. W. Daly (2007), Motion of flux transfer events: A test of the Cooling model, Ann. Geophys., 25, 1669-1690.

Huang, C.-S., A. D. DeJong, and X. Cai (2009), Magnetic flux in the magnetotail and polar cap during sawteeth, isolated substorms, and steady magnetospheric convection events, J. Geophys. Res., 114, A07202, doi:10.1029/2009JA014232.

Kuznetsova, M. M., and L. M. Zeleny (1986), Formation of filaments at the boundaries of magnetospheres, Eur. Space Agency Spec. Publ., ESA SP251, 137-146.

Lee, L. C., and Z. F. Fu (1985), A theory of magnetic flux transfer at the Earth's magnetopause, Geophys. Res. Lett., 12, 105-108, doi:10.1029/ GL012i002p00105.

Lepping, R. P., J. A. Jones, and L. F. Burlaga (1990), Magnetic field structure of interplanetary magnetic clouds at 1 AU, J. Geophys. Res., 95, 11,957-11,965, doi:10.1029/JA095iA08p11957.

Lepping, R. P., D. B. Berdichevsky, C.-C. Wu, A. Szabo, T. Narock, F. Mariani, A. J. Lazarus, and A. J. Quivers (2006), A summary of Wind magnetic clouds for years 1995-2003: Model-fitted parameters, associated errors and classifications, Ann. Geophys., 24, 215-245.

Lundquist, S. (1950), Magnetohydrostatic fields, Ark. Fys., 2, 361-372.

Owen, C. J., A. Marchaudon, M. W. Dunlop, A. N. Fazakerley, J.-M. Bosqued, J. P. Dewhurst, R. C. Fear, S. A. Fuselier, A. Balogh, and H. Rèmy (2008), Cluster observations of "crater" flux transfer events in the dayside high-latitude magnetopause, J. Geophys. Res., 113, A07S04, doi:10.1029/2007JA012701.

Russell, C. T., and R. C. Elphic (1978), Initial ISEE magnetometer results: Magnetopause observations, Space Sci. Rev., 22, 681-715, doi:10.1007/ BF00212619.

Russell, C. T., and R. J. Walker (1985), Flux transfer events at Mercury, J. Geophys. Res., 90, 11,067-11,074, doi:10.1029/JA090iA11p11067.

Sarantos, M., R. M. Killen, and D. Kim (2007), Predicting the long-term solar wind ion-sputtering source at Mercury, Planet. Space Sci., 55 1584-1595, doi:10.1016/j.pss.2006.10.011

Scholer, M. (1988), Magnetic flux transfer at the magnetopause based on single X-line bursty reconnection, Geophys. Res. Lett., 15, 291-294, doi:10.1029/GL015i004p00291.

Slavin, J. A., et al. (2008), Mercury's magnetosphere after MESSENGER's first flyby, Science, 321, 85-89, doi:10.1126/science.1159040.

Slavin, J. A., et al. (2009a), MESSENGER observations of magnetic reconnection in Mercury's magnetosphere, Science, 324, 606-610, doi:10.1126/science. 1172011 . 
Slavin, J. A., et al. (2009b), MESSENGER observations of Mercury's magnetosphere during northward IMF, Geophys. Res. Lett., 36, L02101, doi:10.1029/2008GL036158.

Southwood, D. J., C. J. Farrugia, and M. A. Saunders (1988), What are flux transfer events?, Planet. Space Sci., 36, 503-508, doi:10.1016/00320633(88)90109-2.

Wang, Y. L., et al. (2005), Initial results of high-latitude magnetopause and low-latitude flank flux transfer events from 3 years of Cluster observations, J. Geophys. Res., 110, A11221, doi:10.1029/2005JA011150.

Zhang, H., K. K. Khurana, M. G. Kivelson, V. Angelopoulos, Z. Y. Pu, Q.-G. Zong, J. Liu, and X.-Z. Zhou (2008), Modeling a force-free flux transfer event probed by multiple THEMIS spacecraft, J. Geophys. Res., 113, A00C05, doi:10.1029/2008JA013451.

B. J. Anderson, H. Korth, and R. L. McNutt Jr., Johns Hopkins University Applied Physics Laboratory, Laurel, MD 20723, USA.

D. N. Baker and W. E. McClintock, Laboratory for Atmospheric and Space Physics, University of Colorado, Boulder, CO 80303, USA.
M. Benna and R. M. Killen, Solar System Exploration Division, NASA Goddard Space Flight Center, Greenbelt, MD 20771, USA.

S. A. Boardsen, R. P. Lepping, M. Sarantos, and J. A. Slavin, Heliophysics Science Division, NASA Goddard Space Flight Center, Greenbelt, MD 20771, USA. (james.a.slavin@nasa.gov)

S. M. Krimigis, Johns Hopkins University Applied Physics Laboratory, Laurel, MD 20723, USA.

D. Schriver, Institute for Geophysics and Planetary Physics, University of California, Los Angeles, CA 90024, USA.

S. C. Solomon, Department of Terrestrial Magnetism, Carnegie Institution of Washington, Washington, DC 20015, USA.

P. Trávníček, 11Astronomical Institute, Academy of Sciences of the Czech Republic, CZ-14131 Prague, Czech Republic.

C.-C. Wu, Naval Research Laboratory, Washington, DC 20375, USA.

T. H. Zurbuchen, Department of Atmospheric, Oceanic and Space Sciences, University of Michigan, Ann Arbor, MI 48109, USA. 